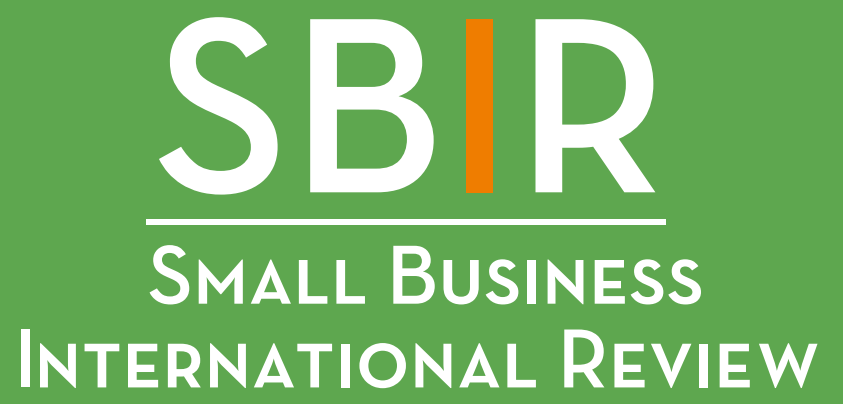

Volumen 1, Número 1

Julio - Diciembre de 2017

pp. 53-70

DOI: https://doi.org/10.26784/sbir.vlii.7

E-ISSN: 2531-0046

Recibido: 2016-09-30

Aprobado: 2017-05-27

\title{
Ventajas competitivas en empresas manufactureras, resultado de los sistemas de producción y la innovación
}

\section{Competitive advantages in manufacturing companies as a result of production systems and innovation}

\section{Salomón Montejano-García}

Profesor investigador, Universidad Autónoma de Aguascalientes, Aguascalientes, México.

Doctor en Ciencias Administrativas, Maestría en Estrategias para Sistemas de Calidad, Universidad Autónoma de Aguascalientes, México. Ingeniero Industrial Mecánico, Instituto Tecnológico de Aguascalientes, México. Integrante del Cuerpo Académico Gestión de las Organizaciones, UAA, Aguascalientes, México.

E-mail: smontej@correo.uaa.mx

\section{Rocío Montserrat Campos-García}

Profesora investigadora, Universidad Autónoma de Aguascalientes, Aguascalientes, México. Doctor en Logística Universidad Autónoma de Querétaro, México. Maestría en Ingeniería Industrial, Instituto Tecnológico de Aguascalientes, México. Ingeniero Industrial, Instituto Tecnológico de Aguascalientes, México. Integrante del Cuerpo Académico Gestión de las Organizaciones, UAA, Aguascalientes, México.

E-mail:rmcampos@correo.uaa.mx

\section{Ricardo García-Ramírez}

Profesor investigador, Universidad Autónoma de Aguascalientes, Aguascalientes, México. Doctor en Ciencias administrativas, UAA, México. Maestría en Administración, Universidad de las Américas, Puebla, México. Contador Público, Escuela Superior de Comercio y Administración, Politécnico de México. Integrante del Cuerpo Académico Gestión de las Organizaciones, UAA, Aguascalientes, México.

E-mail: rgarciar@correo.uaa.mx

\section{Resumen}

Actualmente, es vital para la empresa manufacturera contar con determinadas características, las cuales, logren establecer claramente la diferencia entre éstas y sus similares, por lo tanto es necesario avanzar en el desarrollo de las ventajas competitivas; y así, demostrar cuales son las fortalezas con las que cuenta la empresa, para competir con sus similares y salir adelante sobre éstas, aunque se trate de épocas adversas. Es por ello que la presente investigación, tiene como objetivo, determinar si el desarrollo de ventajas competitivas, tiene alguna relación con la situación en la que se encuentran los sistemas de producción, así como la 
innovación en las empresas manufactureras en Aguascalientes, México. Finalmente el estudio hace notar que en Aguascalientes, en este momento, el desarrollo de ventajas competitivas, se ve influenciado por lo que acontece en los sistemas de producción y la innovación con que cuentan las empresas manufactureras.

Palabras clave: Ventajas competitivas, sistemas de producción, innovación, empresas manufactureras.

\begin{abstract}
Is so important for the manufacture organizations, to get in this moment some special characteristics for clearly establish, differences between them and the competition. So is important to advance in their development, and demonstrate the strength of this company for compete and success; even in adverse times; the objective of this research is, to determine if the development of competitive advantages to have relation, whit the production systems situation as well as the innovation, in manufacturer companies in Aguascalientes, México. This research did show that in this moment, the advantages development, in influenced against the production systems and the innovation in the manufacture factories.
\end{abstract}

Keywords: Competitive advantages, production systems, innovation, manufacturing companies. 


\section{Introducción}

A partir de la revolución industrial el desarrollo de la industria manufacturera se manifestó en el mundo como diferencial de la riqueza entre países, y como resultado el bienestar de sus habitantes; por lo tanto entre más industrializado es un país, sus habitantes se verán igualmente beneficiados. Es responsabilidad de quienes dirigen las industrias, trabajar para que se logre el crecimiento y desarrollo de las mismas (Bunge, 2013); esto es posible si a las empresas que se encuentran actualmente vulnerables a la competencia se les prepara conscientemente para desarrollarse y competir; de esta forma pueden lograr la reducción en costos, una serie de cualidades en las organizaciones para poder hacer diferenciación, entre éstas y otras empresas que sean similares (Porter, 1990), al grupo de características positivas que se logran establecer, se le conoce como ventajas competitivas.

Las empresas manufactureras que pretenden lograr el desarrollo de ventajas competitivas deben preocuparse por aplicar acciones de mejora constantes en sus sistemas de producción, lo cual se logra a través de diferentes técnicas de ingeniería industrial y administración de operaciones, éstas, están encaminadas a lograr la actualización y optimización de los procesos que forman parte de la operación de la empresa. Asimismo se puede hacer mediante la modernización de la tecnología que se dispone para las actividades en el proceso de producción; asimismo se puede recurrir a la gestión de la metodología de mejora continua en las diferentes formas de trabajo que se relacionan al proceso de producción; con este propósito se han desarrollado diferentes metodologías, las cuales, están encaminadas a mejorar de manera constante los métodos de trabajo que realizan durante la operación, así como los procedimientos que se llevan a cabo (Jeh-Nan y Chun-Yi, 2009).

Entre otras formas recomendadas para que las organizaciones desarrollen estas cualidades, reconocidas como ventajas competitivas, se puede recurrir consistentemente, a la aplicación de principios de innovación, que se define como una forma más de influir en la mejora de las organizaciones, con el propósito de establecer en ellas, características propias, éstas les permiti- rán ser diferentes y mejores que su competencia (O'Regan, Ghobadian y Sims, 2006). Por lo tanto, si se desea contar con ventajas competitivas al interior de la empresa, se hace necesario poner en práctica con fortaleza y constancia la innovación en todas sus dimensiones, y de esta manera lograr posicionarse claramente sobre la competencia (Pullen, Weerd-Nederhof, Groen, Song y Fisscher, 2009). En muchas ocasiones, se espera que el resultado del esfuerzo por mejorar el desempeño de la empresa, sea inmediato y muy grande; sin embargo es necesario comprender, que si los cambios son pequeños y graduales estos deben tomarse como resultados satisfactorios, derivados de la aplicación de principios de innovación.

Para el caso particular de este estudio, el Estado de Aguascalientes - México, es correcto afirmar que, si bien no puede considerarse como líder en el país, si cuenta con un gran desarrollo industrial. La anterior situación es desarrollada por Gutiérrez et al. (1999), quienes explican que, durante la década de 1980, llego a la región inversión extranjera para la cual el sistema industrial local no estaba preparado; como resultado, algunas empresas locales desaparecieron, en tanto que otras, emplearon su estructura como maquilas para las empresas foráneas; esta situación se generó por la diferencia palpable entre empresas locales y extranjeras, en el desarrollo de ventajas competitivas. Con esto como ejemplo, se puede ver que el desarrollo de ventajas competitivas, se ha convertido en algo indispensable para que la empresa local mejore y no se vea perjudicada por la competencia; esto solamente se puede lograr si se trabaja con profesionalismo, mediante la aplicación de diferentes técnicas de ingeniería industrial y de administración de operaciones, para permitir que de manera natural las organizaciones locales, se encuentren claramente posicionadas en la preferencia de los clientes

\section{Revisión de la literatura}

Un cuestionamiento presente en el mundo de los negocios, es determinar ¿cómo puede una empresa competir y ser mejor que sus similares? Así como, ¿porqué, algunas empresas aunque pequeñas logran desarrollarse, hasta convertir- 
se en grandes negocios; en tanto que otras, no logran destacar, sino que por el contrario, parece que éstas tienden a desaparecer? La necesidad de respuestas, es ocasionada por la rapidez con que las organizaciones que se encuentran alrededor de las empresas locales, muestran nuevas estructuras y formas de trabajo que les permiten aprovechar las oportunidades para ser altamente competitivas (Brito y Tavares, 2005). Esta situación les pone de manifiesto a las empresas locales, que es necesario que se apliquen consistentemente, para estar a la altura de aquellas que se convierten en su competencia directa; por lo tanto se deben preocupar por estar mejor preparadas y sustentadas en el desarrollo de ventajas competitivas (Sachs, Clifford, Ziness y Eilat, 2000; Schwab y Porter, 2003).

En este sentido, Michel Porter (1990) expresa que para triunfar sobre la competencia es necesario que las organizaciones se ocupen de trabajar fuertemente sobre la reducción de costos, y en el establecimiento de características que hagan patente la diferencia entre las organizaciones y su respectiva competencia. A estas características, que diferencian las organizaciones, se les denomina ventajas competitivas. Cuando se expusieron en un inicio, no se especificaron ante la comunidad académica; sin embargo, con el paso de tiempo se fueron depurando los conceptos de acuerdo a las particularidades del ámbito empresarial y se propusieron ventajas competitivas estratégicas.

Hayes y Schemenner, (1978) y Training Commission (1988), fueron de los primeros investigadores en tratar este tema; definían las ventajas compe- titivas como las estrategias que eran seleccionadas por las compañías, con el objetivo de competir con otras, para cumplir con el objetivo final de generar rendimiento. Pasado el tiempo, se han diseñado estándares para evaluarlas; se definen como la serie de habilidades que influyen directamente en el grado de cumplimiento de los objetivos propuestos, derivados de diversas actividades, una vez que se han realizado con determinado nivel de desempeño, del cual depende el éxito de la organización (Training Commission, 1988, Adam, Everett y Swamidass, 1989).

Actualmente, las ventajas competitivas son consideradas como la serie de cualidades que tiene la organización para apoyarse en el cumplimiento de sus objetivos estratégicos, conformadas por el conjunto de aptitudes, actividades y tecnología que se desarrolla continuamente para obtener mejores resultados que otras empresas. Estas diferencias son las que finalmente impactan sobre el producto o servicio dado a los clientes, quienes pueden evalúan su desempeño, comparándole con la competencia y facilitando, con este ejercicio, la decisión de compra. (Chase, Aquilano y Jacobs, 2000; Gaither y Frazier, 2000; Nahmias, 2007; Pinheiro, Gouvêa y Jan, 2008).

Las ventajas competitivas han sido clasificadas por los investigadores según su intención al fomentar la competitivdad de la industria (Hill, 1985; Miller y Roth, 1988; Porter, 1990; Noori y Radford, 1997; Gaither y Frazier, 2000; Davis, Aquilano y Chase, 2001; Nahmias, 2007; Yong Choon y Ki, 2008; Martín y Díaz, 2009; Lee, 2009), de donde se parte para la creación de estrategias que logren estos aspectos específicos.

Tabla 1. Veces que se mencionan cualidades de la empresa, como ventajas competitivas.

\begin{tabular}{|c|c|c|c|c|c|}
\hline Ventaja competitiva & $n^{\circ}$ & Ventaja competitiva & $n^{\circ}$ & Ventaja competitiva & $n^{\circ}$ \\
\hline $\begin{array}{c}\text { Capacidad para producir e } \\
\text { infraestructura }\end{array}$ & 3 & $\begin{array}{c}\text { Estandarización de métodos } \\
\text { y sistemas }\end{array}$ & 3 & $\begin{array}{l}\text { Estado y disposición de las } \\
\text { instalaciones }\end{array}$ & 1 \\
\hline $\begin{array}{c}\text { Diseño e ingeniería de } \\
\text { producto }\end{array}$ & 3 & Sistemas de distribución & 1 & Retorno de inversión & 2 \\
\hline Cuidado al medio ambiente & 1 & Flexibilidad & 13 & Calidad & 14 \\
\hline $\begin{array}{c}\text { Relación empleados y } \\
\text { enfoque }\end{array}$ & 3 & Servicio postventa & 6 & Costo/precio & 15 \\
\hline Tecnología de procesos & 5 & Integración vertical & 3 & Tiempo de entrega & 11 \\
\hline \multicolumn{6}{|c|}{$\begin{array}{l}\text { Fuente: elaboración propia, basado en Hill, 1985; Miller y Roth, 1988; Porter, 1990; Noori y Radford, 1997; } \\
\text { Gaither y Frazier, 2000; Davis, Aquilano y Chase, 2001; Nahmias, 2007; Yong et al, 2008; Martín y Díaz, } \\
\text { 2009; Lee, } 2009 .\end{array}$} \\
\hline
\end{tabular}


La Tabla 1 muestra la frecuencia con que se menciona en la literatura revisada, las características consideradas como "ventajas competitivas", las cuales que se desarrollan en las organizaciones para competir; de acuerdo a lo anterior las más mencionadas, son reducción de costos, tiempo de entrega, flexibilidad y calidad, por lo que se considerarán éstas para la investigación. Para lograr que dichas cualidades se conviertan en ventajas competitivas, existen una serie de tácticas que se pueden llevar a cabo para realizarlo; sin embargo el presente estudio solo analizará la relación entre ellas y la aplicación de principios de innovación, como también la relación del desarrollo de ventajas competitivas con el estado de los sistemas de producción en empresas manufactureras.

Es necesario exponer, que en un principio, la innovación era considerada solo como soporte para la generación de ideas en la organización, ya que los cambios se dan cuando éstas se realizan; sin embargo a los procesos de innovación no se le da suficiente énfasis; existen estudios que afirman que la innovación se encuentra en evolución constante, por lo tanto nunca se dejará de utilizar, aunque sea con enfoques y formas de trabajo diferentes, pero como su objetivo fundamental, es impactar directamente a la sociedad (Comisión Europea, 1995), una vez que haya apoyado primeramente a la organización.

Dada la competitividad existente a lo largo del mundo, la innovación es reconocida como una de las estrategias principales para sobrevivir y triunfar en el ámbito empresarial, ya que de manera voluntaria o no, la globalización es un fenómeno que actualmente afecta a todos (Pellicer, Yepes y Rojas, 2010), por lo tanto, cada vez son más los investigadores que se suman al análisis de la innovación y a su relación con el desarrollo de ventajas competitivas, como una manera práctica de apoyar a la empresa (Van Lam y Wattanapruttipaisan, 2005; O' Reagan et al. 2006; Cardentey y Quintana, 2008; Yong et al., 2008; Lee, 2009; Pan y Lee, 2011). Actualmente a la innovación, se le considera como una forma práctica para ubicar a la empresa en posición de competir, pues fortalece la existencia de ventajas competitivas, al lograr la aparición de nuevos productos, así como por el progreso en las operaciones, una vez que se aplica la mejora propuesta, en los sistemas de trabajo y los procesos (Van Echtelt et al. 2008).

Asimismo, de acuerdo a la profundidad en la mejora propuesta, la innovación era considerada como una característica en las organizaciones que se aplicaba a grandes cambios y a resultados espectaculares, por lo tanto, los requerimientos para su aplicación, se pensaba que eran también muy especializados (Comisión Europea, 1995), sin embargo, con el transcurrir del tiempo, se admite que no es necesario pensar solamente en grandes cambios, sino que aún las mejoras elementales son consideradas como resultado de la innovación; en el primer caso se habla de innovación radical, y en el segundo, de innovación incremental (Sher y Yang, 2005). La realidad es que de una u otra forma, el término innovación ha trascendido hasta el punto de explicar que se manifiesta en los productos, los procesos, y en los sistemas de gestión, para responder a la generación de ventajas competitivas, así como a la mejora en el ambiente empresarial que rodea a la organización (OCDE y Eurostat, 2005; Van Auken et al. 2008).

No obstante, se reconoce que para algunas empresas es difícil estructurar una ruta clara que les apoye en la aplicación de principios de la innovación, o para el desarrollo de ventajas competitivas, con el objetivo de optimizar la cadena de valor a través del proceso productivo, las cuales se relacionan entre sí, desde la identificación de las necesidades del cliente hasta la distribución de los productos producidos (Luzzini y Ronchi, 2011).

Las empresas manufactureras, de acuerdo al ramo industrial en el que se desenvuelven, están sujetas a diferentes y grandes presiones, causadas por la competencia a la que se enfrentan; por un lado, la influencia de empresas en el orbe, que generan altas posibilidades de nuevos mercados, lo cual causa la reducción del mercado doméstico; esto ocasiona, a los productores locales, que el consumo interno se encuentre en crisis, ya que el arribo de los productores extranjeros motiva que se reduzca la oportunidad de negocio para ellos (Ehrlenspiel, 2003); lo anterior obliga a los empresarios locales a actualizarse al mismo ritmo que lo hace el mundo. Para ello es indispensable modernizar sus sistemas 
Figura 1. Modelo de investigación

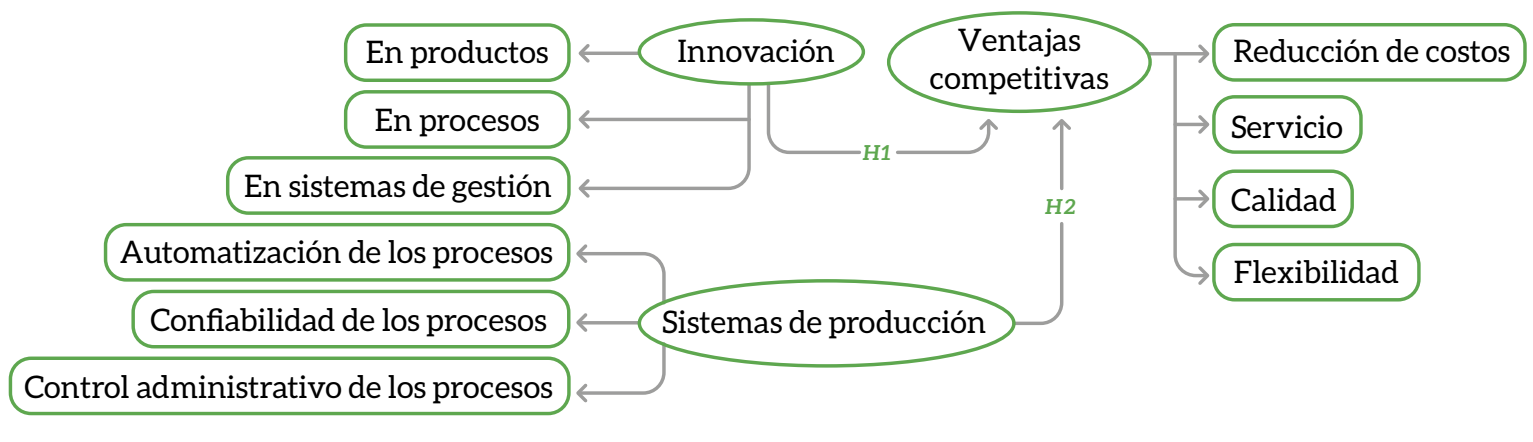

Fuente: elaboración propia.

de producción, pues el uso de tecnologías modernas en los procesos es una diferencia notable entre las empresas locales y extranjeras, estableciendo una ventaja competitiva (Rogalski, 2012).

Es necesario expresar que los sistemas de producción han evolucionado a partir del siglo XIX, y experimentado cambios vertiginosos desde finales del siglo XX, que se reflejan claramente en la automatización, el control administrativo y la confiabilidad de los procesos de producción. Este desarrollo no lo experimentan las empresas, pues su modo de trabajo es tradicional y, al no tener desarrolladas sus ventajas competitivas, no sobreviven a la competencia. (Nahmias, 2007).

Los sistemas de producción se forman por una serie variable de personas, equipo, maquinaria, $\mathrm{y}$ otros elementos, que se combinan de manera ordenada para transformarlos por medio de sistemas y métodos de trabajo hasta obtener un producto (Zhang y Rodríguez, 2009); por lo tanto es indispensable organizar las actividades del proceso, para servir de información a los clientes, y garantizar que hay control de las operaciones requeridas para la elaboración del producto. Por esto el proceso de producción inicia con la identificación de los requerimientos del cliente y termina con su satisfacción, entonces es responsabilidad de la organización, planificar los suministros en forma y tiempo, y determinar la manera de realizar las operaciones involucradas (Azadeh et al. 2008).
La Figura 1 muestra el modelo de estudio, en donde se analiza el comportamiento de la relación entre innovación y ventajas competitivas, así como sistemas de producción y ventajas competitivas de las empresas manufactureras en Aguascalientes.

En cuanto a la relación de innovación con el desarrollo ventajas competitivas algunos estudios teóricos dicen que al desarrollar la innovación, se generan ventajas competitivas como consecuencia del mejoramiento de los procesos, así como por la aplicación de nuevas tecnologías (Cardentey y Quintana, 2008; Arana-Solares et al.2012). A pesar de esto, algunos estudios empíricos realizados para verificar el impacto de la innovación sobre el desarrollo de ventajas competitivas, tienen al ser comparados, resultados controvertidos, ya que, en tanto que un estudio realizado en Corea sobre el impacto de la innovación en productos, sobre el desarrollo de ventajas competitivas resultó no significativo (Yong et al., 2008); en cambio, otro estudio acerca de la innovación en los procesos, indicó que el desarrollo de ventajas competitivas se logra por este medio, especialmente en lo que respecta a calidad (Pan y Lee, 2011).

Algunos teóricos como Van Lam y Wattanapruttipaisan (2005), expresan que el desarrollo de ventajas competitivas se relaciona fuertemente a la innovación, y ésta se puede manifestar para lograr su propósito, ya sea por medio de la inteligencia artificial como fuente de desarrollo de nuevos productos, por la mejora de los procesos, o por la gestión del conocimiento. O' Reagan et 
al. (2006), expresan que para lograr el desarrollo de ventajas competitivas, se debe utilizar la innovación. Por su lado, Cardentey y Quintana (2008), mencionan que también reconocen a la innovación como una práctica importante para que las empresas desarrollen e incrementen las ventajas competitivas; especialmente, ellos hacen mención especial a la reducción de costos.

La investigación empírica se hace presente, ya que basado en lo expresado anteriormente por los teóricos en materia, se realizaron algunas pruebas empíricas a este respecto por parte de algunos investigadores; los resultados empíricos que se obtuvieron, fueron diferentes y no todos de acuerdo con la teoría. A este respecto, Yong et al (2008), en Corea, realizó un análisis de correlación entre la aplicación de innovación y el desarrollo de ventajas competitivas, el resultado indicó, que esta relación es no significativa; por lo tanto en este estudio, no se aceptó que exista influencia de la innovación sobre el desarrollo de ventajas competitivas. Se realizó un estudio similar pero en otra locación, Lee (2009), concluyó que efectivamente, existe influencia significativa de la innovación sobre el desarrollo de ventajas competitivas. Posteriormente, Pan y Lee (2011) realizaron pruebas de control de algunos procesos no conformes; después de aplicar técnicas de mejora lograron dejarlos muy cerca del límite central, concluyendo que la aplicación de innovación incremental apoya el desarrollo de ventajas competitivas, especialmente la calidad. Con base en lo anterior se establece la siguiente hipótesis.

H1: La aplicación de principios de innovación influye significativamente en el desarrollo de ventajas competitivas.

La relación de sistemas de producción y desarrollo de ventajas competitivas también ha sido estudiada teórica y empíricamente por diferentes investigadores; algunos de ellos a finales del siglo XX dictaron sus conclusiones teóricas a este respecto (Coch y French, 1948; Miles y Snow, 1978, 1981; Skinner, 1978; Swing y Hegarty, 1998). Por otro lado con el inicio del siglo XXI, con base en las conclusiones teóricas hechas con anterioridad, diferentes investigadores realizaron pruebas empíricas para determinar la relación de los sistemas de producción con el desarrollo de ventajas competitivas (Aris et al. 2000; Brito y Tavares, 2005; Urgal, 2007; Yong et al., 2008).

Las conclusiones teóricas de algunos investigadores, (Coch y French, 1948; Miles y Snow, 1978, 1981), explican que la división del trabajo, así como la especialización, se convierten en conceptos vitales para poder mejorar los sistemas de producción, los cuales cuando tienden a la optimización, facilitan el desarrollo la flexibilidad de los mismos, además de apoyar en la calidad, y en la reducción de costos, entre otras ventajas competitivas. Asimismo, Skinner (1978), explica que en los sistemas de producción se pueden realizar una serie de mejoras que se reflejan de inmediato en ellos mismos; en el incremento en la organización, métodos de pago, prácticas de supervisión, control de producción y diseño del trabajo, entre otros; estos a su vez, generan ventajas competitivas como la calidad, la reducción de costos, etc. Posteriormente, Swing y Hegarty (1998), mencionan que, a causa de las capacidades por la infraestructura de manufactura, se logran desarrollar habilidades en las áreas de proceso, que a su vez, permiten diferenciar a sus productos respecto de sus competidores. Asimismo, Pan y Lee (2011), hacen mención a que, además de innovar, es necesario la aplicación de técnicas de administración de operaciones y de control de los procesos de producción para generar ventajas competitivas.

En cuanto los estudios empíricos de la relación entre los sistemas de producción y el desarrollo de ventajas competitivas, Aris et al. (2000), expresan que la principal razón para incluir la utilización de tecnología avanzada en los sistemas de producción, es mejorar la calidad, incrementar la productividad y ser competitivos en costos, entre otras. En este mismo sentido, Brito y Tavares (2005), después de un estudio descriptivo, concluyen que efectivamente, existe relación directa entre la flexibilización de los sistemas de producción con el desarrollo de ventajas competitivas. Después de un análisis de regresión jerárquica, Urgal (2007), concluye que las cuatro dimensiones resultantes de este análisis, impactan positivamente sobre cinco dimensiones que resultan del análisis del desarrollo de las ventajas competitivas. Contrariamente, después de hacer un estudio en Corea, se generaron 
hipótesis para probar el efecto de los sistemas de producción sobre el desarrollo de ventajas competitivasYong et al (2008), concluye que dicha relación no es significativa por lo que opina que no hay influencia de los sistemas de producción sobre el desarrollo de ventajas competitivas. Del análisis realizado, se desprende la siguiente hipótesis:

H2: La actualización de los sistemas de producción, influye significativamente en el desarrollo de las ventajas competitivas.

\section{Metodología}

En Aguascalientes se tiene registro de la existencia de 5.638 empresas manufactureras (Directorio estadístico nacional de unidades económicas, INEGI 2010), sin embargo la presente investigación se desarrollara con el objetivo de analizar a las empresas manufactureras de Aguascalientes que tienen una cantidad de empleados superior a diez. De acuerdo a la información analizada, Aguascalientense cuenta con 490 empresas manufactureras; en la tabla 2, se tiene la clasificación por tamaño de las empresas, de acuerdo al número de empleados.

Tabla 2. Clasificación de la industria manufacturera por tamaño en Aguascalientes.

\begin{tabular}{ccc}
\hline Número de empleados & Número de empresas & $\%$ \\
\hline $11-30$ & 294 & 60 \\
$31-50$ & 60 & 12.24 \\
51100 & 51 & 10.41 \\
101250 & 37 & 7.55 \\
$251-$ más & 48 & 9.80 \\
Total & 490 & 100.00 \\
\hline
\end{tabular}

Fuente: elaboración propia basado en INEGI (2010).

El estudio es transversal, no experimental y cuantitativo; para obtener información se diseñó una encuesta con tres apartados para conocer la opinión de 300 empresarios en la ciudad de Aguascalientes, México, y determinar la manera en que estos promueven en sus empresas principios de innovación, como describen los sistemas de producción, así como el desarrollo de ventajas competitivas, en sus respectivas firmas. Se cuenta con un total de 49 preguntas likert 5, las cuales se califican como se describe en la Tabla 3.

\begin{tabular}{ll|c}
\hline \multicolumn{2}{c}{ Tabla 3. } & Descripción de calificación según escala Likert 5 \\
\hline \multicolumn{1}{c}{ Constructo analizado } & \multicolumn{1}{c}{ Dimensiones } & Interpretación de resultados \\
\hline Sistemas de producción & Automatización de procesos & \\
& Confiabilidad de procesos & 1 Nunca se aplican \\
& Control administrativo & 2 Ocasionalmente se aplican \\
Innovación & Productos & 3 Normalmente se emplean \\
& Procesos & 4 Casi siempre se da aplicación \\
& Sistemas de gestión & 5 Siempre se les da aplicación \\
Ventajas competitivas & Costos, Calidad, Flexibilidad, & \\
& Entrega &
\end{tabular}

Fuente: elaboración propia.

Para evaluar el constructo "Sistemas de producción" se utilizó una escala de 20 variables, adaptada por Martínez et al (2010), y por Maldonado et al. (2011), asimismo, se consultó a otros autores como Raymond y St-Pierre (2005), Urgal (2007), según se muestra en Tabla 4. 
Tabla 4. Descripción de variables constructo sistemas de producción

\section{Variables por constructo}

1.- Se cuenta con procesos de producción automatizados.

2.- La maquinaria que se utiliza, cuenta con algún tipo de software.

3.- Se cuenta con maquinaria controlada por PLC's.

4.- Se cuenta con equipo mecánico tradicional.

5.- Se cuenta con maquinaria controlada por control numérico.

6.- Se cuenta con controles de calidad automatizados.

7.- Se cuenta con registros formales de productividad.

8.- Se cuenta con plan maestro de producción.

9.- Cuenta con tecnología, la cual en su mayoría es extranjera.

10.- Cuenta con tecnología desarrollada por la propia empresa.

11.- Cuenta con tecnología, la cual es menor a a diez años.

12.- Se cuenta con procesos de operación flexibles.

13.- Se opera con una capacidad de producción superior al $50 \%$

14.-Cuenta con un control estadístico del proceso de producción

15.- Cuenta con una carta de control de procesos.

16.- Cuenta con un programa de mantenimiento productivo total.

17.- Cuenta con un programa de mantenimiento preventivo.

18.- Cuenta con un sistema de control de calidad.

19.- Cuenta con un control de los insumos que requiere la producción

20.- Cuenta con una bitácora de mantenimiento de la maquinaria y el equipo.

Fuente: elaboración propia.

Para el constructo innovación, se utilizó una escala de 12 preguntas, según se expresa en la Tabla 5, fue probada por Van Auken et al. (2008); y utilizada localmente por Martínez et al. (2010) y por Mojica (2012).

Tabla 5. Descripción de variables constructo innovación

\section{Variables por constructo}

1.- La modificación a productos y el diseño de nuevos productos generados que se realizan por año.

2.- La inversión de recursos que se realiza para introducir nuevos productos o productos nuevos al mercado es.

3.- Se introducen nuevos productos al mercado, más rápidamente que nuestros competidores.

4.-Se hace inversión en investigación y desarrollo de nuevos productos.

5.- La cantidad de modificaciones que se realizan en los procesos por año es.

6.- Se realiza inversión de recursos para la adopción de nuevos procesos o para mejorar de los procesos actuales.

7.- La integración de nuevos procesos en la empresa, se hace de manera más rápida que nuestros competidores.

8.- Se realizan inversiones para el desarrollo de nuevos procesos y mejoras en los procesos actuales.

9.- El número de ocasiones en que se realizan cambios o mejoras en los sistemas administrativos al año es.

10.- El grado de novedad que tienen los sistemas administrativos de la empresa.

11.- La investigación que se hace sobre nuevos sistemas administrativos por parte de los ejecutivos de la empresa es.

12.- La inversión de recursos que se realiza para la introducción de nuevos procesos administrativos o mejoras

Fuente: elaboración propia.

En el caso de ventajas competitivas, se adaptó una escala con base en la información proporcionada por diferentes autores como Henderson's (1979), Crosby (1979), Porter's (1990), Hambrick's (1983), Deming (1985), Jurán (1993), Noori y Radford (1997), Britro y Tavares (2005), Faria et al. (2005), Urgal, (2007), Roig et al. (2007), Pinheiro de Lima et al, (2008), Karaulova et al.(2009), según se expresa en la Tabla 6. 
Tabla 6. Descripción de variables constructo ventajas competitivas

Variables

1.- Se cuenta con sistemas y métodos de control formales, que apoyan en el control de costos.

2.- Se ponen en práctica programas y métodos confiables para la reducción de los costos en materiales

3.- Se tiene identificado que acciones se pueden realizar para controlar los costos en el proceso productivo.

4.- Se cuenta con sistemas de determinación y control de costos estándar

5.- El área de manufactura, fabrica productos que no tiene ningún defecto.

6.- La habilidad de fabricar un producto que cumpla con las especificaciones del diseño se manifiesta

7.- El producto que se fabrica, trabaja de acuerdo al tiempo esperado por la empresa.

8.- Se tiene la posibilidad de ofrecer un servicio personalizado de acuerdo a las necesidades de los clientes.

9.- Se cuenta con la habilidad para incrementar la producción ante aumentos no previstos en la demanda.

19.- Se cuenta con la capacidad de cambiar el tamaño de los lotes de fabricación sin afectar calidad y costo.

11.- Se tiene la facilidad para introducir en la producción, cambios rápidos derivados del diseño del producto, sin afectar el flujo del proceso.

12.- Se cuenta con la facilidad de producir una gran gama de productos, sin la necesidad de hacer grandes cambios en el proceso.

13.- Se tiene la capacidad para ajustar rápidamente y con costos mínimos la fabricación de varios tipos de productos al mismo tiempo.

14.- Se cuenta con la capacidad de ofrecer cualquier pedido rápidamente y sin problemas o sobre costo.

15.- Se tiene la facilidad para entregar los productos en el preciso momento en que el cliente los solicite.

16.- Se cuenta con la facilidad para atender las devoluciones sin afectar al cliente.

17.- Se cuenta con disponibilidad para atender cualquier cambio de volumen en el memento que sea solicitado por el cliente

Fuente: elaboración propia.

Los resultados obtenidos de las pruebas de fiabilidad se presentan en la Tabla 7. Se observa que después de las pruebas de las 49 variables del modelo teórico, solo quedaron 42 para formar el modelo de investigación, ya que, únicamente éstas cumplen con la pertinencia en el análisis, por tener un Alpha de Cronbach superior en todos los casos a 0.7 (Nunnally y Bernstein, 1994; Lévy y Varela, 2006); y contar con el índice de fiabilidad compuesta (IFC), con valor superior a 0.7 además, los valores resultantes del análisis de varianza extraída (IVE), cumplen al tener un valor superior a 0.5 (Fornell y Larcker, 1981; Ping, 2004); por lo tanto se considera que existe fiabilidad en el nuevo modelo ajustado.

\begin{tabular}{|c|c|c|c|c|c|c|c|c|c|c|}
\hline \multirow[b]{2}{*}{ Constructo } & \multirow[b]{2}{*}{$\begin{array}{l}\text { Alphade } \\
\text { Cronbach }\end{array}$} & \multirow[b]{2}{*}{ Dimensión } & \multicolumn{3}{|c|}{ Modelo teórico } & \multicolumn{3}{|c|}{ Modelo ajustado } & \multirow[b]{2}{*}{ IFC } & \multirow[b]{2}{*}{ IVE } \\
\hline & & & variables & $\begin{array}{l}\text { Alpha de } \\
\text { Cronbach }\end{array}$ & IFC & IVE & variables & $\begin{array}{l}\text { Alpha de } \\
\text { Cronbach }\end{array}$ & & \\
\hline \multirow{2}{*}{$\begin{array}{l}\text { Procesos de } \\
\text { producción }\end{array}$} & \multirow{2}{*}{0.908} & Automatización & 6 & 0.816 & 0.830 & 0.488 & 5 & 0.875 & 0.875 & 0.585 \\
\hline & & Confiabilidad & 7 & 0.797 & 0.784 & 0.360 & 4 & 0.776 & 0.793 & 0.504 \\
\hline \multirow{3}{*}{ Innovación } & \multirow{3}{*}{0.943} & $\begin{array}{l}\text { Control } \\
\text { administrativo. }\end{array}$ & 7 & 0.893 & 0.893 & 0.546 & 7 & 0.893 & 0.893 & 0.545 \\
\hline & & Productos & 4 & 0.885 & 0.887 & 0.662 & 4 & 0.885 & 0.887 & 0.662 \\
\hline & & Procesos & 4 & 0.887 & 0.891 & 0.672 & 4 & 0.887 & 0.891 & 0.672 \\
\hline \multirow[t]{5}{*}{$\begin{array}{l}\text { Ventajas } \\
\text { competitivas }\end{array}$} & \multirow[t]{5}{*}{ s 0.794} & $\begin{array}{l}\text { Sistemas } \\
\text { gestión }\end{array}$ & 4 & 0.872 & 0.876 & 0.639 & 4 & 0.872 & 0.876 & 0.639 \\
\hline & & Costos & 4 & 0.820 & 0.821 & 0.542 & 4 & 0.820 & 0.821 & 0.542 \\
\hline & & Calidad & 4 & 0.780 & 0.789 & 0.489 & 3 & 0.774 & 0.790 & 0.563 \\
\hline & & Flexibilidad & 5 & 0.818 & 0.822 & 0.482 & 4 & 0.806 & 0.815 & 0.527 \\
\hline & & Tiempo entrega & 4 & 0.628 & 0.721 & 0.410 & 3 & 0.740 & 0.752 & 0.506 \\
\hline Global & 0.927 & Total & 49 & & & & 42 & & & \\
\hline
\end{tabular}

Fuente: elaboración propia basado en los resultados de pruebas de fiabilidad de los modelos teórico y ajustado. 


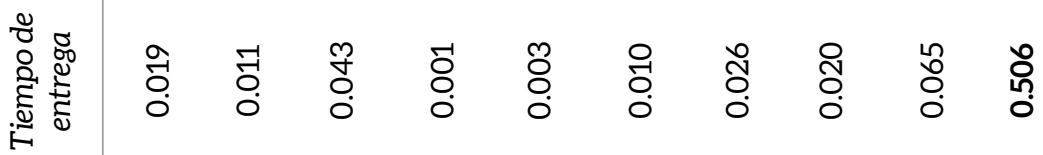

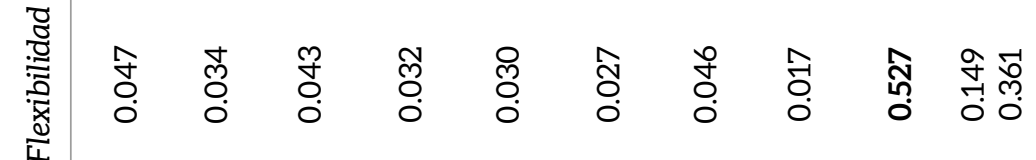

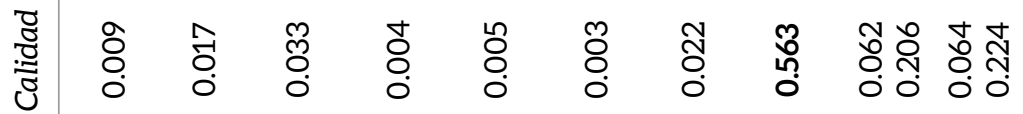

苞

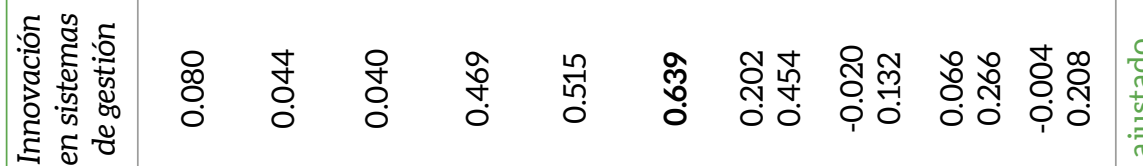

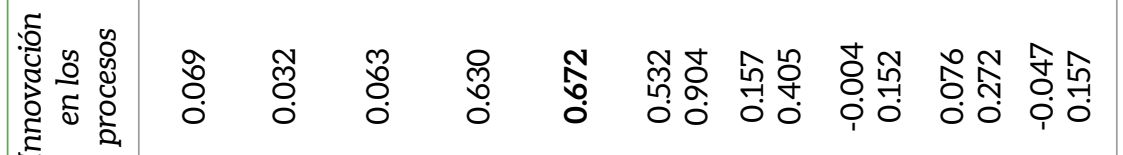

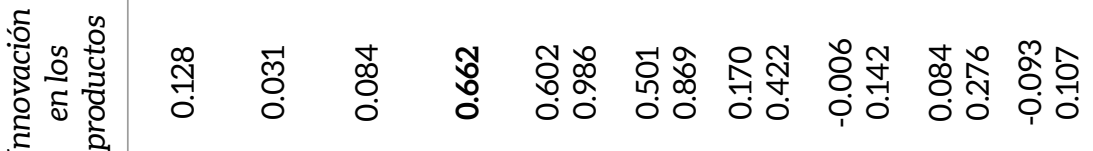

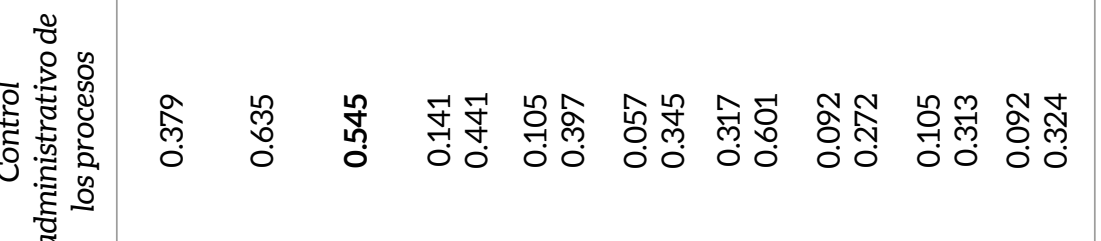

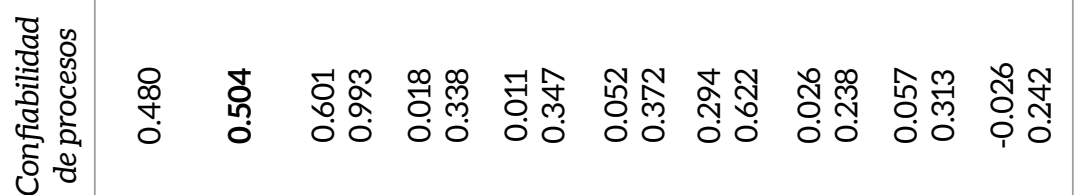

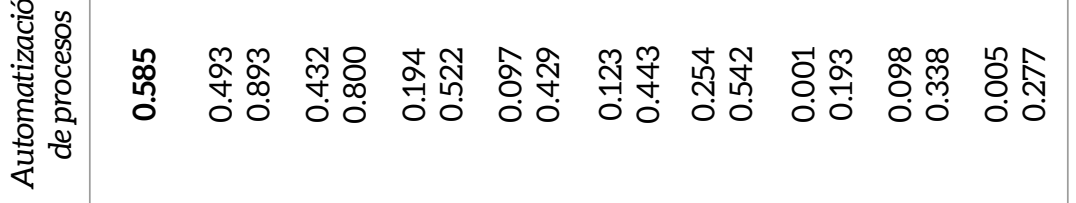

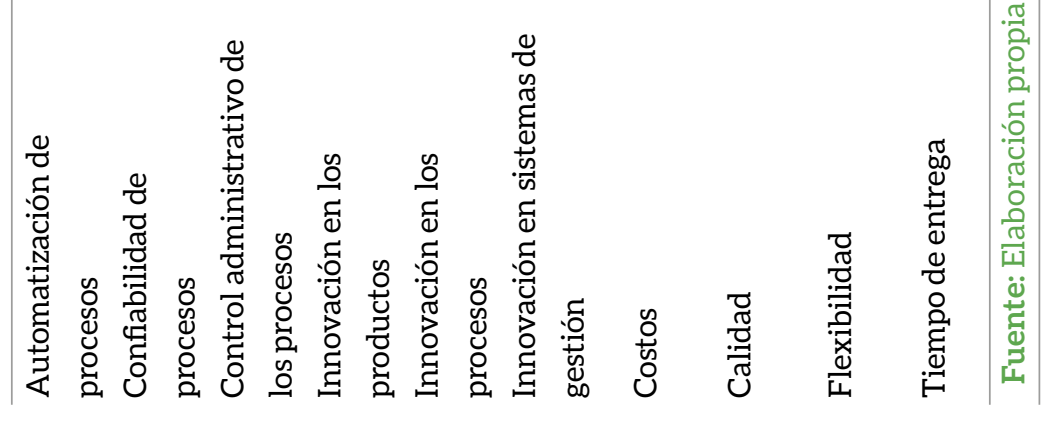


Por último la Tabla 8 muestra los resultados de la prueba de validez, donde se puede ver en la parte inferior a la diagonal, que en ninguno de los casos se encuentra la cantidad uno entre los limites expresados, por lo tanto las covarianzas de cada una de las variables relacionadas es diferente, y se expresa que no miden lo mismo, sino que cada una de ellas mide lo propio; asimismo, en la parte superior se expresa el resultado de la prueba de varianza extraída, en ella los valores expresados son inferiores a los del índice de la varianza extraída, a excepción en la que relaciona confiabilidad de productos con el control administrativo de los procesos, la cual es ligeramente superior; así, la validez del modelo se confirma.

Asimismo, para la realización de los análisis estadísticos necesarios se utilizó regresión lineal múltiple, y para apoyar su realización, se acudió a los paquetes estadísticos, SPSS-19 y el EQS- 6.1. Inicialmente, para verificar que las escalas utilizadas contaban con la pertinencia suficiente para ser utilizadas en las conclusiones del estudio realizado, se llevaron a cabo, las pruebas de fiabilidad y validez requeridas para el modelo inicial de investigación.

\section{Resultados}

Una vez determinadas la fiabilidad y validez del modelo de investigación, se procede a la realización de los análisis estadísticos. En ellos se observa que el empresario en Aguascalientes, no aplica formalmente técnicas para mejorar sus sistemas de producción, y únicamente cuando observa la necesidad de algún cambio, procede a hacer las correcciones necesarias. En este sentido, se puede ver que la dimensión automatización de los procesos es la que menor atención tiene, en cambio el control administrativo de los procesos es lo que mayormente ocupa su atención; esta situación se hace patente, principalmente en las empresas pequeñas.

En cuanto a la innovación, se observa que es una de las acciones que el empresario en Aguascalientes practica frecuentemente; en este momento, manifiesta mayor interés por ésta que por los sistemas de producción. La innovación se refleja con mayor fortaleza en los procesos y en los productos, que en los sistemas de gestión. Las empresas grandes realizan éstas tareas con mayor formalidad y constancia que las medianas y pequeñas.

En lo que respecta al desarrollo de ventajas competitivas, el empresario en Aguascalientes afirma que frecuentemente está realizando tareas que se relacionan con el perfeccionamiento de éstas, en este momento, las consideradas de mayor importancia son la flexibilidad y el tiempo de entrega. Posiblemente, esto se debe a la reducción de inventarios que existe en las empresas transnacionales, derivado de la filosofía justo a tiempo, que priva en las organizaciones actuales. Por otra parte, de las ventajas competitivas analizadas, la reducción de costos es a la que menor atención se le presta.

La Tabla 9, muestra los resultados del análisis de correlación para el modelo de investigación. En primer lugar, se puede ver que para la relación entre la aplicación de innovación y el desarrollo de ventajas competitivas, la variable independiente es innovación y la dependiente el desarrollo de ventajas competitivas; de manera que con carga factorial de 0.346 y con $\mathrm{R}^{2}$ de 0.096 se determina que los cambios en las ventajas competitivas, se explican en $9.6 \%$ por los cambios ocurridos en la innovación de las empresas; los valores de $t$, explican que la influencia de la innovación sobre las ventajas competitivas es significativa a un $95 \%$, en este sentido, la primera de las hipótesis planteadas en el estudio no se rechaza.

Por lo tanto, para en las empresas manufactureras en Aguascalientes, al incrementar la innovación se tiene como resultado, el incremento en el desarrollo de ventajas competitivas.

En segundo lugar, se expresa el resultado de la relación entre los sistemas de producción y el desarrollo de ventajas competitivas; en este análisis, se consideró variable independiente a los sistemas de producción y como variable dependiente las ventajas competitivas; la carga factorial de la variable sistemas de producción es 0.463 sobre el desarrollo de ventajas competitivas, con $\mathrm{R}^{2}$ de 0.193 ; por lo tanto, se determina que el cambio en las ventajas competitivas se explica en $19.3 \%$ por el cambio en los siste- 
Tabla 9. Resultado de la regresión lineal entre innovación y ventajas competitivas

\begin{tabular}{|c|c|c|c|c|}
\hline Hipótesis & Relación & Valor de $\beta$ & $R 2$ & $t$ \\
\hline $\begin{array}{l}\text { H1: La aplicación de principios de } \\
\text { innovación en las empresas } \\
\text { manufactureras en Aguascalientes, } \\
\text { influye significativamente en el } \\
\text { desarrollo de ventajas competitivas. }\end{array}$ & $\begin{array}{c}\text { Innovación } \\
\text { Ventajas competitivas }\end{array}$ & $0.346^{*}$ & 0.096 & 2.24 \\
\hline $\begin{array}{l}\text { H2: La actualización de los sistemas de } \\
\text { producción en las empresas } \\
\text { manufactureras en Aguascalientes, } \\
\text { influye significativamente en el } \\
\text { desarrollo de las ventajas competitivas. }\end{array}$ & Ventajas competitivas & $0.463^{* *}$ & 0.193 & 3.18 \\
\hline
\end{tabular}

mas de producción; el valor de t, explica que la influencia es significativa al $99 \%$, por lo que no se rechaza la segunda hipótesis. En este sentido, se dice para Aguascalientes; que si los sistemas de producción en las empresas manufactureras tienen alguna variación, el desarrollo de ventajas competitivas, sufrirán un cambio en el mismo sentido que estos.

\section{Discusión y conclusiones}

El desarrollo de ventajas competitivas es resultado de la forma diferente y acertada en que realizan las tareas en la empresa. Los empresarios en Aguascalientes hacen mención de su preocupación por esta situación, ya que frecuentemente las tienen presentes durante sus operaciones; siendo principalmente la flexibilidad y el tiempo de entrega las de mayor atención, posiblemente, por tratarse de empresas alimentadoras de las transnacionales instaladas en este lugar. Por lo anterior, cambian de procesos y se deben adherir a metodologías de trabajo relacionados con justo a tiempo y manufactura esbelta.

Para lograr hacer realidad esta situación, es necesario, que se reconozca el efecto de la aplicación de innovación sobre el desarrollo de las ventajas competitivas, y comprender que si se mejora en la primera se tendrán efectos positivos sobre la segunda. Por lo tanto, se puede ver que la innovación es una actividad muy importante para el empresario en Aguascalientes.
Este muestra preocupación por innovar, sin embargo para los empresarios locales, muestra preferencia por la innovación en los procesos y en los productos, quizá motivado por la presión en el abasto a empresas transnacionales, de las que son proveedores. Sin embargo, su interés es menor por la innovación en los sistemas de gestión, posiblemente porque las mejoras que se realizan son para resolver los problemas que se presentan durante la etapa de fabricación, para dar cumplimiento a tiempos de entrega. Por lo tanto no se innova de manera sistemática.

De manera, que para que la innovación impacte efectivamente en la generación de ventajas competitivas, es necesario que los empresarios en Aguascalientes trabajen sistemáticamente en las tres dimensiones de la innovación; pero sobre todo, no accionar solamente para resolver problemas emergentes, sino actuar para prevenirlos. Para ello, se recomienda la aplicación de las metodologías de mejora, que se practican en empresas transnacionales; algunas son el despliegue de las funciones de calidad, principios de manufactura esbelta y desarrollo de equipos de mejora; asimismo es necesario, aplicar tecnologías de información en la operación de la empresa para mejorar consistentemente los sistemas de gestión de la misma y apoyar a la operación por medio de sistemas de planeación de los recursos a través de la empresa (ERP), los cuales tienen gran influencia en el control logístico de la cadena de valor, para mejorar básicamente los tiempos de entrega ( Chen 2016). 
En cuanto a los sistemas de producción, el empresario en Aguascalientes, aunque considera que normalmente realiza tareas encaminadas a mejorarlos, la atención a éstos, es menor a la que muestra por la aplicación de innovación en sus organizaciones. No obstante, de acuerdo con la literatura analizada a este respecto, las tareas realizadas por ellos, afectan directamente al desarrollo de ventajas competitivas, especialmente en lo que respecta a la reducción de costos, posiblemente por ser esta diferenciación la que los pone en condición de penetrar en el mercado de servicios de manufactura, mantenimiento y ventas (Urgal, 2007); que son condiciones del mercado en Aguascalientes en este momento, por la influencia de la presencia de empresas extranjeras en la región (Gutiérrez, 2016).

Los resultados de la investigación afirman que al mostrar preocupación por los sistemas de producción; mediante la aplicación de diferentes técnicas o metodologías, se logra pasar de un estado de ventajas competitivas limitadas, a otro en el que se tienen mayores ventajas competitivas que nuestros competidores; pero si nunca mejoran los sistemas de producción, el resultado que se puede obtener es que las ventajas competitivas que se tienen sean si acaso, similares a las de la competencia.

En este sentido, se tiene que el empresario local tiene interés especial por las tareas relacionadas con la confiabilidad de los procesos, sobre todo, lo que tiene que ver con gestión de la calidad y el control administrativo de los procesos. Esto, quizá sea un reflejo del arribo de empresas transnacionales a la región, ya que en este momento representan localmente el liderazgo industrial, al emplear metodologías como justo a tiempo, manufactura esbelta, seis sigma, entre otras; por lo tanto los empresarios locales se ven obligados a implementar estas formas de trabajo, ya que como proveedores deben mejorar constantemente en estos temas (Kamaruddin et al. 2016).

Contrario a esto, las acciones referentes a la automatización de los procesos, son las que se llevan a cabo con menor frecuencia en las empresas en Aguascalientes; quizá por el alto costo que esto significa para quienes las realizan, así como por los bajos volúmenes de producción, los cuales no apoyan la viabilidad de automatizar algunas operaciones; por tal razón, para lograr mejorar las operaciones, sin que esto signifique un gasto, se propone el empleo de técnicas básicas de ingeniería industrial, como son el análisis de métodos, estudios hombre máquina y análisis de la operación (Bas et al. 2016), los cuales pretenden mejorar los procesos de operación vía optimización de las operaciones; y así, con el ahorro obtenido, financiar la automatización de los mismos a gran escala.

\section{Referencias}

Adam, E., Everett. Jr., \& Swamidass, M.P. (1989). Assessing operations management a strategic perspective. Journal of $\mathrm{Ma}$ nagement, 15 (2), 181-203. http://dx.doi. org/10.1177/014920638901500204

Arana-Solares, I.A., Alfalla-Luque, R., y Machuca, J.A.D. (2012). Análisis de las variables que proporcionan una competitividad sostenible en la cadena de suministro. Intangible Capital, 8 (1), 92 - 122. http://dx.doi.org/10.3926/ic.255

Aris, S.S., Raghunathan, T.S., \& Kunnathar, A. (2000). Factors affecting the adoption manufacturing technologies in small firms. Advanced Management Journal, 65(2), 14 - 21. http:// search.proquest.com/openview/683b5fccd313951bb695e6a8e46a2d5d/1?pq-origsite =gs-

cholar\&cbl=40946

Azadeh, A., Haghnevis, M., \& Khodadadegan, Y. (2008). Design of integrated information system, business and production process by simulation. Journal of the American Society for Information Science and Technology, 59 (2), 216 - 234. http://dx.doi.org/10.1002/asi.20712

Bas, G., Stoev, L., \& Durakbasa, N.M. (2016). Assessment of the production quality in machining by integrating a system of high precision measurement. Procedia Engeniering. 100, 1616 - 1624. https://doi.org/10.1016/j. proeng.2015.01.535

Brito, S. R.O., \& Tavares D.P.R. (2005). Alignament of management priorities, manufac- 
turing flexibility and performance in small companies, Brazilian Journal of Operations \& Production Management, 2(1), 57-80. Recovered 03/06/2017 https://bjopm.emnuvens.com.br// bjopm/article/view/BJV2N1_2005_P4

Bunge, M. (2013). Filosofía Política, Solidaridad, Cooperación y Democracia Integral. Madrid, España: Gedisa, $1^{\mathrm{a}} \mathrm{Ed}$.

Cardentey, A.I.M. y Quintana, M.O. (2008). Propuesta de metodología para elaborar la estrategia de gestión de la innovación tecnológica en las empresas cubanas. ACIMED, 18(6), 1 -16. Recuperado 03/06/2017 http://scielo.sld.cu/pdf/ aci/v18n6/aci101208.pdf

Chase, R.B., y Aquilano N.J. y Jacobs. F.R. (2000). Administración de producción y operaciones. Bogotá, Colombia: Mc. Graw Hill, $8^{a}$ Ed.

Chen, J. (2016). Sourcing for quality: Cooperating with a single supplier or developing two competing suppliers? Hindawi $\mathrm{Pu}$ blishing Corporation, 1, 1 - 13. http://dx.doi. org/10.1155/2016/3040343

Coch, L., \& French, J.R. P. (1948). Overcoming resistance to change. Human Relations, 11, 512 - 532. http://journals.sagepub.com/doi/ abs/10.1177/001872674800100408?journalCode=huma

Comisión Europea. (1995). Libro verde de la innovación. Bruselas, Bélgica: Unión Europea. Diciembre.

Crosby, P. (1979). Quality is free. New York, USA: Mc Graw Hill.

Davis, M.M., Aquilano, N.J., y Chase, R.B. (2001). Fundamentos de Dirección de Operaciones (3 ed.). Madrid, España: Mc. Graw Hill.

Deming, W. E. (1985). Transformation of western style of management. Interfaces,15(3), 6 11. http://dx.doi.org/10.1287/inte.15.3.6

Ehrlenspiel, K. (2003). Integrierte produktenwickllung: denkabläufe, methodeneisatz, zusamme- narbeit. Munich, Germany: Karl Hanser Verlag.

Faria, F.F.C., Godinho, F.M., \& Booney, M. (2005). Integrating materials flow, production control and quality control: A proposal and case study. Brazilean Journal of Operations \& Production Management, 2(1), 81 - 104. Recovered 03/06/2017 https://bjopm.emnuvens.com.br// bjopm/article/view/BJV2N1_2005_P5

Fornell, C., \& Larcker, D.F. (1981). Evaluating structural equation models with unobservable variables and measurement error. Journal of Marketing Research, 18(1), 39-50. http://dx.doi. org $/ 10.2307 / 3151312$

Gaither, N., y Frazier, G. (2000). Administración de producción y operaciones (ed. 8). México D.F., México: Editorial Thomson Editores.

Gutiérrez, C.D., Hernández, R.M., y Alemán, L.R. (1999). Nuevas estrategias de modernización empresarial en Aguascalientes (ed. 1). Aguascalientes, México: Universidad Autónoma de Aguascalientes.

Gutiérrez, C.P. (2016). Política pública y relaciones industriales de aglomeración industrial en Aguascalientes, México. México D. F., México: Plaza y Valdés Editores.

Hambrick, D.C. (1983). High profit strategies in maturate capital goods industries: a contingency approach. Academy of Management Journal, 26(4), 687 - 707. http://dx.doi. org/10.2307/255916

Hayes, H.R., \& Schemenner, W.R. (1978). How should you organize manufacturing? Harvard business review, January-February, 204 - 233. Recovered15/06/2017 https://hbr.org/1978/01/ how-should-you-organize-manufacturing

Henderson, B. (1979). Henderson on Corporate Strategy. Cambridge (MA), USA: Abt Books.

Hill, T. (1985). Manufacturing strategy: The strategic management of the manufacturing function. London, UK: Mac Millan.

Jeh-Nan, P., \& Chun-Yi, L. (2009). Development a new process incapability index with 
an application to the evaluation of manufacturing risk. Communication in Statistics Theory and Methods. 38, 1133 - 1153. http://dx.doi. org/10.1080/03610920802220777

Kamaruddin, S.S., Mohammad, M. F., \& Mahbub, R. (2016). Barriers and impact of mechanization in construction to achieve better quality products. Procedia - Social and Behavioral Sciences, 222 (1), 111 - 120. https://doi.org/10.1016/j. sbspro.2016.05.197

Karaulova, T., Shevtshenko, E., Polyanchikov, I., \& Sahno, J. (2009). Reorganisation of production system on SME enterprises. Annals of DAAAM for 2009 \& Proceedings of the 20th International DAAAM 20(1), 869 - 870. Recuperado 15/06/2017 https://www.researchgate.net/profile/Eduard_Shevtshenko/ publication/287589449_Reorganisation_of production_system_on_sme_enterprises/ links/56a87bde08ae860e025658f1.pdf

Lee, S.H. (2009). Developing hierarchical structure for assessing the impact of innovation factors on a firm's competitiveness a dynamic capabilities approach. The Journal of American Academy of Business, Cambridge, 15 (1), 216 223. Recovered 15/06/2017 http://www.jaabc. com/jaabcv15n1preview.html

Lévy, J.P., y Varela, J. (2006). Modelización con estructuras de covarianzas en ciencias Sociales. Temas esenciales, avanzados y aportaciones especiales (ed. 1). A Coruña, España: Netbiblo.

Luzzini, D., \& Ronchi, S. (2011). Organizing the purchasing department for innovation. Operation Management Research, 4, 14 - 27. https:// doi.org/10.1007/s12063-010-0042-2

Maldonado, G.G., Martínez, S.M.C., Hernández, C.O., y García, P.L.D. (2011). El impacto de los procesos de producción en el rendimiento de la Pyme manufacturera de México: Un estudio empírico. TEC Empresarial, 5(1), 21 - 30. Recuperado 15/06/2017 https://dialnet.unirioja.es/ servlet/articulo?codigo=3619099

Martín, P.M.L., y Díaz, G.E. (2009). Posicionamiento estratégico de las empresas industriales en las prioridades competitivas de operaciones:
Desarrollo y aplicación de un indicador de medida. Cuadernos de Economía y Dirección de Empresas, 12(39), 59 - 94. https://doi.org/10.1016/ S1138-5758(09)70035-0

Martínez, S.M.C., García, P.L.D., y Maldonado, G.G. (2010). Innovación y gestión del conocimiento, en la Pyme de Aguascalientes. Aguascalientes, México: Universidad Autónoma de Aguascalientes.

Miles, R.E., \& Snow, C.C. (1978). Organization strategy, structure and process. New York, USA: Mc Graw Hill.

Miles, R.E., \& Snow, C.C. (1981). Toward a synthesis in organization theory. In M. Jelinek, J.A. Litterer, \& R.E. Miles (Eds). Organization by design: Theory and practice, Plano (TX), USA: BPI. $548-562$.

Miller, J.G., \& Roth, A. (1988). Manufacturing strategies. Executive summary of the 1988 manufacturing futures survey. (Manufacturing roundtable research report). Boston (MA), USA: University, Boston.

Mojica, C.E.P. (2012). La influencia d la orientación a mercado y la innovación en la capacidad de internacionalización y desempeño de las PYMES en el estado de Aguascalientes. (Tesis inédita de doctorado). Universidad Autónoma de Aguascalientes, Aguascalientes, México.

Nahmias, S. (2007). Análisis de la Producción y las Operaciones (ed. 5). México D.F., México: McGraw Hill.

Noori, H., y Radford, R. (1997). Administración de Operaciones y Producción (ed. 1). Bogotá, Colombia: Mc. Graw Hill.

Nunnally, J.C., \& Bernstein, I.H. (1994). Psychometric Theory. New York, USA: McGraw Hill.

OCDE y Eurostat. (2005). La medida de las actividades científicas y tecnológicas Manual de OSLO, Guia para la recogida e interpretación de datos sobre innovación (ed. 3). http://www.itq.edu.mx/ convocatorias/manualdeoslo.pdf

Pan, J.N., \& Lee C.Y. (2011). Development of 
a new process incapability index with an application to the evaluation of manufacturing risk. Communication in Statistics Theory and Methods, 5(38), 1133 - 1153. http://dx.doi. org/10.1080/03610920802220777

Pellicer, E., Yepes, V., \& Rojas, R.J. (2010). Innovation and competitiveness in construction companies. Journal of Management Research, 10 (2), 103 - 115. Recovered 03/06/2017 http://search. proquest.com/openview/3371a37632480813fc2685efeec82626/1?pq-origsite $=$ gscholar\&c$\mathrm{bl}=55395$

Ping, R.A. (2004). On assuring valid measures for theoretical models using survey data. Journal of Business Research, l (57), 125-141. https:// doi.org/10.1016/S0148-2963(01)00297-1

Pinheiro de Lima, E., Gouvêa da Costa, S.E., \& Jan Angelis, J. (2008). Framing operations and performance strategic management systems design process. Brazilian Journal of Operations \& Production Management, 5 (1), 23-46. Recovered 15/06/2017 https://www.researchgate.net/ publication/46189776_Framing_Operations and_Performance_Strategic_Management_ System_Design_Process

Porter, M. (1990). The Advantage Competitive of Nation. New York, USA: The free press.

Pullen, A., Weerd-Nedrhof, P., Groen, A., Song, M., \& Fisscher, O. (2009). Successful Patterns of internal SME characteristics leading to high overall innovation performance. Creativity and Innovation Management, 3 (18), 209 - 223. https://doi.org/10.1111/j.1467-8691.2009.00530.x

Raymond, L., \& St-Pierre, J. (2005). Antecedents and performance outcomes of advanced manufacturing systems sophistication in SMEs. International journal of Operations \& Production Management, 25(5/6), 514 - 533. http://dx.doi. org/10.1108/01443570510599692

Rogalski, S. (2012). Factory design and process optimization with flexibility measurements in industrial production. International Journal of Production Research, 50 (21), 6060 - 6071. http:// dx.doi.org/10.1080/00207543.2011.643831
Roig,Z.J.F., Forradelas, R.Q.y Camargo, M.(2007). Multiproduct supply chain - Strategic planning and forecasting. Brazilian journal of operations \& production management, 4 (1), 61 - 87. Recovered 03/06/2017 https://bjopm.emnuvens.com. br//bjopm/article/view/BJV4N1_2007_P4

Sachs, J., Clifford, Z.C. y Eliat, Y. (2000). Benchmarking competitiveness in transition economies. Harvard Institute for International Development, 62, 5 - 18. http://onlinelibrary.wiley. com/doi/10.1111/1468-0351.00078/full

Schwab, K., \& Porter, M. (2003). The global competitiveness Report: 2002-2003. World economic forum Geneva, Switzerland 2003. New York USA: Oxford University press.

Sher, P.J., \& Yang, P.Y. (2005). The effects of innovative capabilities and $R \& D$ clustrewring in firm performance: The evidence of Taiwan's semiconductor industry, Technovation, 25(1), 33 - 43. https://doi.org/10.1016/S01664972(03)00068-3

Skinner, C.W. (1978). Manufacturing in the corporate strategy. New York, USA: Wiley.

Swing, M., \& Hegarty, W.H. (1998). Core capabilities and their links to product differentiation. International Journal of Operations \& Production Management, 18(4), 374 -396. http://dx.doi. org/10.1108/01443579810199748

Training Commission. (1988). Classifying the components of management competences Sheffield, South Yorkshire, UK: Training Commission.

Urgal, G.B. (2007). ¿Cómo influye la infraestructura de producción en el rendimiento de las empresas manufactureras. Cuaderno de Gestión, 7(2), 13 - 27. Recuperado 03/06/2017 https:// core.ac.uk/download/pdf/6565142.pdf

Van Auken, H. Madrid, G. A., \& García, P. de L.D. (2008). Innovation and performance in spanish manufacturing SME's. International Journal of Entrepreneurship and Innovation Management, 8 (1), 36 - 56. https://doi.org/10.1504/ IJEIM.2008.018611 
Van Echtelt, F.E.A., Wynstra, F. ,Van Weele A.J., \& Duysters, G. (2008). Managing supplier involvement into new product development: A multiple case study. Journal Production Innovation Management. 25, 180 - 201. https://doi. org/10.1111/j.1540-5885.2008.00293.x

Van, L.N., \& Wattanapruttipaisan, T. (2005). From Grain.sized innovations to triple-test patents in Asean. Patterns, issues, and implications in development and competitiveness. ASAEAN Economic Bulletin, 22(2), 117 - 143. http://www. jstor.org/stable/25773850?seq=1\#page_scan_ tab_contents

Yong, J.Ch., Choon, S.L., \& Ki, T.S. (2008). The relationships among manufacturing innovation, competitiveness, and business performance in the manufacturing industries of Korea. The International Journal of Advanced Manufacturing Technology, 38(7), 840 - 850. https://doi. org/10.1007/s00170-007-1107-8

Zhang, L.L., y Rodriguez, B. (2009). A tree unification approach to constructing generic process. iee Transactions. 41, 916 - 929. http://dx. doi.org/10.1080/07408170903026049 